\title{
НАСЛЕДСТВЕННЫЕ ФАКТОРЫ РИСКА РАЗВИТИЯ МИОМЫ МАТКИ: ПОИСК МАРКЕРНЫХ ОДНОНУКЛЕОТИДНЫХ ПОЛИМОРФИЗМОВ
}

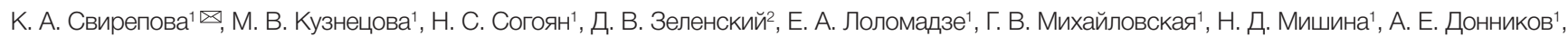
Д. Ю. Тросимов

${ }^{1}$ Национальный медицинский исследовательский центр акушерства, гинекологии и перинатологии имени В. И. Кулакова, Москва, Россия

2 Валуйская центральная районная больница, Валуйки, Россия

Миома матки является одной из важнейших социально значимых проблем женского репродуктивного здоровья. Целью исследования было найти маркерные однонуклеотидные полиморфизмы (SNP), ассоциированные с развитием миомы матки. Для проверки гипотезы о том, что наследственность играет важную роль в патогенезе миом, группу из 100 пациенток с подтвержденным диагнозом миомы матки разделили на две подгруппы: подгруппу Іа с отягощенным семейным анамнезом, подгруппу Іб с неотягощенным семейным анамнезом по миоме матки. Группа сравнения была сформирована из 30 пациенток (женщины в постменопаузе, не имевшие в анамнезе миому матки, отрицавшие наличие миом у ближайших родственниц). Первичную нуклеотидную последовательность определяли с помощью секвенирования по методу Сэнгера. Были выявлены статистически значимые (p < 0,05) различия между исследованными группами по частотам генотипов по rs12637801 и rs12457644. Впервые обнаружены «протективные» SNP, редкие аллели которых могут служить маркерами пониженного риска развития лейомиом матки.

Ключевые слова: миома матки, соматические мутации, однонуклеотидные полиморфизмы

Финансирование: работа выполнена в рамках госзадания 2019 г. «Совершенствование тактики ведения больных доброкачественными заболеваниями органов репродуктивной системы с использованием высокотехнологичных методов функциональной визуальной диагностики и панели молекулярно-биологических маркеров прогрессирования и рецидива заболеваний».

Вклад авторов: К. А. Свирепова - анализ литературы, проведение исследования, написание текста статьи; М. В. Кузнецова - проведение исследования, написание текста статьи; Н. С. Согоян - сбор материала и ведение коллекции в биобанке; Д. В. Зеленский - сбор и предоставление материала для исследования; Е. А. Лоломадзе, Г. В. Михайловская - помощь в проведении лабораторной части исследования; Н. Д. Мишина статистическая обработка результатов; А. Е. Донников, Д. Ю. Трофимов - общее руководство и редактирование статьи.

Соблюдение этических стандартов: исследование одобрено этическим комитетом Национального медицинского исследовательского центра акушерства, гинекологии и перинатологии имени В. И. Кулакова. Все пациентки подписали добровольное инсормированное согласие на участие в исследовании.

$\bigotimes$ Для корреспонденции: Ксения Александровна Свирепова ул. Академика Опарина, д. 4, г. Москва, 117997; kseswi@yandex.ru

Статья получена: 05.02.2020 Статья принята к печати: 19.02.2020 Опубликована онлайн: 28.02.2020 DOI: 10.24075 /vrgmu.2020.011

\section{HEREDITARY RISK FACTORS FOR UTERINE LEIOMYOMA: A SEARCH FOR MARKER SNPS}

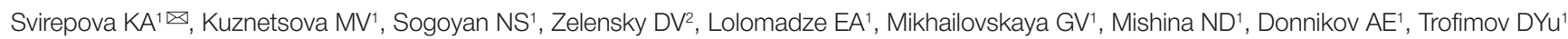

${ }^{1}$ Kulakov National Medical Research Center for Obstetrics, Gynecology and Perinatology, Moscow, Russia

2 Valuyki Central Hospital, Valuyki, Russia

Uterine leiomyomas are a worrying reproductive health issue that has serious social implications. The aim of this study was to conduct a search for marker single nucleotide polymorphisms (SNPs) associated with uterine leiomyoma. To test the hypothesis about the contribution of genetic predisposition to the pathogenesis of myomas, the initial group of 100 patients with a verified diagnosis of uterine leiomyoma was divided into 2 subgroups: subgroup la (women with a family history of the disease) and subgroup $1 \mathrm{~b}$ (women with no family history of the disease). The control group consisted of 30 postmenopausal patients who did not have a medical history of uterine fibroids and denied uterine fibroids in their close female relatives. DNA sequences were read using Sanger sequencing. Statistically significant differences $(p<0.05)$ were discovered between the analyzed groups in terms of genotype frequencies for rs 12637801 and rs 12457644 . Also, previously unknown protective SNPs were identified whose rare alleles could predict the reduced risk of uterine leiomyomas.

Keywords: leiomyoma, somatic mutation, single nucleotide polymorphism

Funding: this study was part of the State Assignment (2019) on the Improved management of patients with benign reproductive system neoplasms with hi-tech diagnostic imaging techniques and molecular panels for predicting the progression and relapse of the disease.

Author contribution: Svirepova KA analyzed the literature, carried out the research and wrote the manuscript with input from all authors; Kuznetsova MV carried out the research and wrote the manuscript with input from all authors; Sogoyan NS collected tissue samples and documented them in the biobank's register; Zelensky DV collected tissue samples for research; Lolomadze EA, Mikhailovskaya GV helped with the laboratory part of the research; Mishina ND performed statistical analysis; Donnikov AE, Trofimov DYu supervised the study and revised the manuscript.

Compliance with ethical standards: the study was approved by the Ethics Committee of Kulakov National Medical Research Center for Obstetrics, Gynecology and Perinatology. Informed consent was obtained from all participants.

$\triangle$ Correspondence should be addressed: Ksenia A. Svirepova

Akademika Oparina, 4, Moscow, 117997; kseswi@yandex.ru

Received: 05.02.2020 Accepted: 19.02.2020 Published online: 28.02.2020

DOI: $10.24075 /$ brsmu.2020.011

Лейомиома матки - одна из наиболее распространенных доброкачественных опухолей женской половой системы [1]. Среди женщин репродуктивного возраста частота ее встречаемости составляет 40-50\%, у одной трети пациенток развитие таких опухолей сопровождается серьезными симптомами [1-4].
Миома матки зачастую протекает бессимптомно, особенно в начале развития заболевания, при небольших размерах узлов, их небольшом количестве. Однако существуют тревожные симптомы, которые могут стать причиной значительного снижения качества жизни у большей части женского населения. Основные жалобы 
пациенток связаны с болевым синдромом при растущей миоме; утомляемостью, слабостью, рассеянностью, появляющимися на фоне менометроррагии и хронической анемии; диспареунией, психологическим стрессом, связанным с вышеуказанными проблемами и страхом перед возможными медицинскими вмешательствами или перед нарушениями репродуктивной функции [5-8].

Важную роль данной гинекологической патологии отводят и в качестве причины женского бесплодия. При обследовании по поводу как первичного, так и вторичного бесплодия миому матки обнаруживают в 23,5\% случаев [9]. В мире лейомиома ассоциирована с 10\% случаев бесплодия у женщин и является единственной причиной бесплодия у 1-3\% пациенток. Влияние миомы матки на бесплодие в значительной степени зависит от локализации миоматозных узлов [10].

Социальная значимость данного заболевания также высока по ряду причин. Современная женщина ведет активный образ жизни, и симптомы, связанные с ростом миомы, могут существенно ухудшать качество жизни женщины, снижать ее работоспособность, увеличивать частоту госпитализаций в гинекологические стационары, в связи с этим увеличиваются затраты государства на лечение таких пациенток.

Научная литература содержит большой объем информации, посвященной поискам фракторов, вызывающих развитие миомы матки, среди которых генетические, гормональные и ряд других. Однако однозначных причинноследственных отношений между этими факторами и патогенезом миом пока не установлено. Согласно популяционным исследованиям распространенности заболевания, важнейшую роль в развитии миом играет генетическая предрасположенность - примерно у 5-10\% женщин наблюдается "семейная форма» миомы матки [1]. Опубликован также ряд исследований, показывающих, что для женщин афро-американского происхождения характерен повышенный риск развития лейомиомы, что демонстрирует генетические различия между расами в отношении рисков развития заболевания. Согласно современным данным, в возрасте 50 лет примерно у 70-80\% женщин обнаруживают хотя бы один миоматозный узел, а развитие тяжелых осложнений возможно почти у 15-30\% больных [11-14].

Уже более 20 лет активно идут поиски генетических маркеров развития лейомиом. Так как миомы являются моноклональными опухолями, т. е. развиваются из одной клетки-предшественницы, то с появлением NGS-методов соматические изменения генома миом стали изучать довольно интенсивно. Было установлено, что наиболее частым вариантом соматических изменений генома в лейомиомах становятся мутации в гене MED12, большая часть из которых представляет собой однонуклеотидные замены в кодонах 43 и 44 экзона 2 [15]. Данный вид соматических мутаций выявлен в 70\% случаев. Это стало наиболее значимым открытием, связанным с патогенезом миом, поскольку в миомах с мутацией в MED12 не обнаружили больше никаких соматических изменений генома. Кроме того, было показано, что в миомах с мутацией в MED12 возрастает экспрессия гена RAD51B, что также может способствовать клеточной пролиферации и росту опухоли [16-17]. Ген MED12 расположен на X-хромосоме и кодирует белок массой 250 кДа, представляющий собой субъединицу большого медиаторного комплекса и участвующего в регуляции транскрипции комплекса PHKполимеразы II.
При дальнейшем изучении было установлено, что в гене MED12 наиболее распространена соматическая мутация $131 \mathrm{G}>\mathrm{A}$. Всего в экзоне 2 гена MED12 удалось выявить шесть вариантов однонуклеотидных замен В трех позициях: 130G $>A, 130 G>C, 130 G>T, 131 G>T$, $130 \mathrm{G}>\mathrm{T}, 131 \mathrm{G}>\mathrm{C}$ (расположены по увеличению частоты встречаемости) [18].

Несмотря на высокую распространенность мутаций MED12 в лейомиомах матки человека, причины их возникновения и механизм действия до сих пор неизвестны. В опубликованном нашей группой в 2016 г. исследовании было выявлено, что соматические мутации в экзоне 2 гена MED12 обнаруживали в 50\% образцов миоматозных узлов среди исследованной выборки российских женщин [19-20]. Перспективным представляется дальнейший поиск генетических маркеров, ассоциированных с риском развития заболеваний. Такой подход уже используют при изучении целого ряда онкологических заболеваний. Так, для эндометриоза уже выявлены однонуклеотидные полиморфизмы (SNP), наличие патогенных аллелей которых повышает риск развития этого заболевания [21].

Поиск генетических маркеров развития лейомимы идет во все мире. В метаанализе, проведенном в 2020 г. [22], продемонстрирована прямая связь девяти SNP с риском развития миомы матки на уровне значимости всего генома $\left(p<6,6 \cdot 10^{-9}\right)$ : rs3820282 (1p36.12), rs124793436 (2p25.1), rs2251795 (3q26.2), rs2242652 (5p15.33), rs75228775 (10q24.33), rs2280543 (11p15.5), rs17033114 (12q23.2), rs7989971 (13q14.11) и rs12484776 (22q13.1) в популяции японских женщин. При этом rs2251795, rs2242652, rs75228775, rs2280543 и rs7989971 показали статистически значимые эффекты у пациенток с множественными миоматозными узлами по сравнению с пациентками с одиночным миоматозным узлом. Два SNP (rs2251795 и rs75228775) были ассоциированы с субмукозной лейомиомой, a rs2280543 на 11 p15.5 был связан с интрамуральной лейомиомой матки. Данные ассоциации подчеркивают важность дальнейших исследований в этой области с целью выявить влияние вариантов аллелей различных генов на патогенез столь распространенного и до конца непонятного заболевания, как лейомиома матки.

Цель исследования заключалась в поиске маркерных SNP развития лейомиомы матки.

\section{ПАЦИЕНТЫ И МЕТОДЫ}

Отбор пациентов для проведения исследования осуществляли в отделении оперативной гинекологии Научного центра акушерства, гинекологии и перинатологии имени В. И. Кулакова и в гинекологическом отделении Валуйской центральной районной больницы в период с 2018-2019 гт. В исследование были включены 100 пациенток с диагнозом миомы матки. Критерии включения пациенток в исследование: репродуктивный возраст; наличие миомы матки, подтвержденной клинически и функциональными методами исследования (для основной группы); показания к оперативному лечению. Критерии исключения пациенток: острые инфекционные заболевания; аденомиоз III, IV степени; злокачественные заболевания органов малого таза; противопоказания к оперативному вмешательству. Для проверки гипотезы о том, что наследственность играет важную роль в патогенезе миом, основную группу, состоящую из 100 пациенток с подтвержденным диагнозом миомы матки, разделили на две подгруппы: в подгруппу la 
вошли 53 пациентки в возрасте 20-46 лет с отягощенным анамнезом, у которых миома была диагностирована у ближайших родственниц по материнской линии (мамы, бабушки, сестры, тети) с единичными или множественными миоматозными узлами; в подгруппу lб вошло 47 пациенток в возрасте 19-42 лет с неотягощенным анамнезом по миоме матки с единичными или множественными миоматозными узлами. Выделение данных подгрупп проводили на стадии опроса и сбора анамнеза пациенток. Группа сравнения была сформирована из 30 пациенток (женщины в постменопаузе, не имевшие в анамнезе миому матки, отрицавшие наличие миом у ближайших родственниц).

\section{Отбор образцов}

Сбор образцов тканей миом производили непосредственно во время операций миомэктомии или гистероэктомии (объем операций зависел от размера и количества узлов). Фрагменты тканей помещали в физиологический раствор, отправляли в биобанк и замораживали при $-70{ }^{\circ} \mathrm{C}$ для последующего хранения в коллекции. Образцы каждого узла подвергали гистологическому исследованию с целью подтверждения наличия исключительно ткани миоматозного узла в образце и отсутствия в нем ткани капсулы или миометрия.

\section{Выделение ДНК}

ДНК выделяли с помощью набора QIAamp DNA Blood Mini Kit (Qiagen; США) согласно протоколу производителя.

\section{Постановка ПЦР-реакции}

ПЦР-реакции проводили в термоциклере S1000тм (BioRad: США) при следующих температурных режимах: начальная денатурация в течение 2 мин при $94^{\circ} \mathrm{C}$, далее 35 циклов с последовательной сменой температур: денатурация при $94{ }^{\circ} \mathrm{C}$ в течение 60 с, отжиг праймера при $64^{\circ} \mathrm{C}-60$ c, элонгация при $72{ }^{\circ} \mathrm{C}-1,5$ мин, конечная стадия $72{ }^{\circ} \mathrm{C}-$ 10 мин (табл. 1). Эффективность амплификации определяли с помощью электрофореза в 2\%-м агарозном геле. Гели окрашивали бромистым этидием. Для визуализации и документирования результатов электросореза применяли систему гель-документирования ChemiDoc XRS+ (Bio-Rad ChemiDoc XRS Gel Photo Documentation System) (Bio-Rad; США).

\section{Постановка сиквенсной реакции}

Первичную нуклеотидную последовательность определяли секвенированием по методу Сэнгера. ПЦР-реакцию для секвенирования проводили с помощью набора Big Dye X-terminator v. 1.1 (Applied Biosystems; CШA). Полученные ПЦР-продукты анализировали на приборе ABI PRISM 3130 Genetic Analyzer (Applied Biosystems; США). Последовательности ампликонов сопоставляли с соответствующими референсными последовательностями по каждому rs. При анализе последовательностей использовали программный пакет BioEdit (Tom Hall; CША).

\section{Метод прямого переосаждения ДНК в мягких условиях}

Очищенный ПЦР-продукт получали методом прямого осаждения ДНК в мягких условиях для очистки смеси от праймеров и других компонентов ПЦР-реакции, без использования коммерческих продуктов. Применяли смесь $\mathrm{NH}_{4} \mathrm{Ac}+\mathrm{EtOH}$ (конечная концентрация ацетата аммония 0,125 М, этанола - 70\%). В каждую из пробирок с ПЦРпродуктом (10 мкл ПЦР-смеси) добавляли 50 мкл смеси ацетата аммония с этанолом, перемешивали на вортексе или переворачиванием. Высаживание проводили при комнатной температуре в течение 20 мин, затем центрифугировали при 13000 об./мин в течение 15 мин, удаляли супернатант, осадок промывали 100 мкл 70\%-го этанола комнатной температуры, центрифугировали еще 15 мин при 13000 об./мин, удаляли супенатант и высушивали в термостате или вакуумной центрифуге, после этого добавляли в каждую пробирку по 20 мкл формамида.

\section{Обработка полученных результатов в редакторе BioEdit}

На основании полученных сиквенсных хроматограмм были определены генотипы пяти исследуемых полиморфизмов для изучаемых образцов в редакторе выравнивания биологических последовательностей BioEdit (Tom Hall; США).

\section{Статистическая обработка данных}

Для проведения статистического анализа данных использовали следующие программные продукты: Microsoft Excel 2013 (Microsoft; США), библиотеки SciPy (SciPy 1.4.1, Python Software Foundation; CША), Pandas для

Таблица 1. Олигонуклеотидные праймеры для секвенирования участков генома, в которых локализованы исследуемые полиморфизмы

\begin{tabular}{|c|c|c|}
\hline ID & Ген & Нуклеотидная последовательность 5' $\rightarrow 3^{\prime}$ \\
\hline \multirow{2}{*}{ rs12637801 } & \multirow{2}{*}{ KCNMB2 } & s3: p-5'-CCA Tgg gCT ACA gTT TAC CA-3' \\
\hline & & a4: p-5'-gTC CCT gTA AgA ATg CTT ggA C-3' \\
\hline \multirow{2}{*}{ rs2861221 } & \multirow{2}{*}{ CELF4 } & s1: 5'-gCC СТC TgT gCT Cgg gAA -3' \\
\hline & & a2: 5'-Tgg CCC AgC AgT gAT AAA gT -3' \\
\hline \multirow{2}{*}{ rs3020434 } & \multirow{2}{*}{ ESR1 } & s1: 5'-TTg CgC TTT gCT gTT AAT gAA g -3' \\
\hline & & a2: 5'-TgA CCC TAA TAC ACC TAg gAA AgT g -3' \\
\hline \multirow{2}{*}{ rs11742635 } & \multirow{2}{*}{ FBN2 } & s1: 5'-ATC CAA ATA gTg AAA ACT Cag Tag gTA C -3' \\
\hline & & a2: 5'-gTg gAg CAT CAg TTA TAg gAA ggC -3' \\
\hline \multirow{2}{*}{ rs12457644 } & \multirow{2}{*}{ CELF4 } & s3: p-5'-TAC ggg CAg ACA ACg ggT-3' \\
\hline & & a4: p-5'-AAg CCC TTg gTA TTC TAg CCT TAC-3' \\
\hline
\end{tabular}


языка программирования Python 2.7 (pandas 1.0.1., Wes McKinney; США). Для оценки характера распределения количественных данных предварительно проводили тест с W-критерием Шапиро-Уилка. Поскольку в большинстве случаев распределение данных было отлично от нормального, использовали методы непараметрической статистики. В качестве меры центральной тенденции количественных признаков была выбрана медиана (Ме), а в качестве интервальной оценки - верхний $(H)$ и нижний квартили (L). Результаты представляли в виде $\mathrm{Me}(\mathrm{L}-\mathrm{H})$. Для оценки значимости межгрупповых различий применяли U-критерий Манна-Уитни для несвязанных совокупностей. Достоверность различий в частоте встречаемости качественных признаков определяли по критерию $\chi^{2}$ с поправкой на правдоподобие. Оценку соответствия выявленных частот генотипов закону Харди-Вайнберга проводили по критерию $\chi^{2}$ в сравнении с ожидаемыми частотами генотипов равновесного распределения. Статистически значимыми считали различия при $p<0,05$ (95\%-й уровень значимости). Отношение шансов (ОШ) представлено с 95\%-м доверительным интервалом (ДИ). При оценке ассоциации аллеля с фенотипическим признаком (наличие миомы матки и наследственная отягощенность анамнеза по этому заболеванию) сравнивали генотипические частоты анализируемого аллеля в группах пациенток, имеющих и не имеющих данный признак. При наличии статистически значимых различий в распределении аллелей сравнивали распределение генотипов в данных группах. При этом проверяли гипотезы об аутосомно-доминантном и аутосомно-рецессивном типе наследования анализируемого признака методом построения соответствующих модели четырехпольных таблиц. Для каждой таблицы рассчитывали двухсторонний критерий Фишера $(F)$ при количестве наблюдений в одной из ячеек менее пяти или критерий $\chi^{2}$. Также рассчитывали отношение шансов (ОШ) проявления признака при соответствующем генотипе (гомозиготы при аутосомнорецессивном типе наследования и гетеро- и гомозиготы для аутосомно-доминантного). ОШ приведено с 95\%-м доверительным интервалом (ДИ). 95\%-е доверительные интервалы рассчитывали на основании распределения $\chi^{2}$, как описано в Statistical Methods for Rates and Proportions [23]. Заключение о наиболее вероятном типе наследования делали по результатам сравнения моделей: наилучшей признавали модель с максимальной статистической значимостью различий в распределении генотипов.

\section{РЕЗУЛЬТАТЫ ИССЛЕДОВАНИЯ}

Генотипирование образцов проводили по следующим локусам: KCNMB2 - rs12637801, CELF4 — rs2861221, ESR1 - rs3020434, FBN2 - rs11742635, CELF4 rs12457644. Данные SNP были выбраны на основании пилотного исследования, проведенного с помощью микроматричного анализа на чипах SNP 6.0 (Thermofisher; США) для 20 пациенток с отягощенным семейным анамнезом по миоме матки и наличием соматических мутаций в гене MED12 и 14 пациенток контрольной группы (женщины в постменопаузе, не имевшие в анамнезе миому матки). С помощью статистического анализа было проведено сопоставление частот 906600 SNP между группами, после чего шесть кандидатных SNP, для которых выявили различия в распределении аллелей и генотипов, были выбраны для генотипирования на более широкой выборке из 100 пациенток с миомами (табл. 2).

Был проведен анализ ассоциации между наличием заболевания/наследственной отягощенностью анамнеза и распределением аллелей исследуемого полиморфизма. Распределение частот генотипов для всех исследованных полиморфизмов соответствовало закону Харди-Вайнберга. Для оценки ассоциации генотипа пациентки с миомой матки были проанализированы распределения генотипов исследуемых полиморфизмов среди трех групп пациенток (табл. 3, 4).

При сопоставлении группы сравнения (данная группа представлена меньшей выборкой по сравнению с основной группой женщин, имеющих миому матки) с подгруппой la (пациенток с отягощенным анамнезом по миоме матки), была обнаружена статистически значимая разница в частоте выявления «протективных» вариантов полиморфизмов и аллелей рисков развития миомы матки, что указывает на возможность использовать данные полиморфизмы в качестве маркеров для прогнозирования вероятности развития миомы. Увеличение частот минорных аллелей в группе сравнения в сопоставлении с группой женщин с миомами гораздо достовернее выявлено в подгруппе больных с отягощенным семейным анамнезом, что свидетельствует о роли генетической компоненты в развитии наследственных, “семейных» форм лейомиоматоза.

Статистический анализ результатов, приведенных в табл. 4, показал, что частота распространенного аллеля $\mathrm{G}$ однонуклеотидного полиморфизма rs11742635 достоверно

Таблица 2. «Кандидатные» полиморфизмы и гены и места их локализации

\begin{tabular}{|c|c|c|c|c|}
\hline ID & Ген & Мутации/замены & Название последовательности & Координаты в геноме (g.) согласно базе HGVS \\
\hline rs12637801 & KCNMB2 & $C>A$ & $\begin{array}{l}\text { GRCh37.p13 chr } 3 \\
\text { GRCh38.p12 chr } 3\end{array}$ & $\begin{array}{l}\text { NC_000003.11:g.178379500C }>A \\
\text { NC_000003.12:g.178661712C>A }\end{array}$ \\
\hline rs2861221 & CELF4 & $C>G$ & $\begin{array}{l}\text { GRCh37.p13 chr } 18 \\
\text { GRCh38.p12 chr } 18\end{array}$ & $\begin{array}{l}\text { NC_000018.9:g.34940179 C>G } \\
\text { NC_000018.10:g.37360216 C>G }\end{array}$ \\
\hline rs3020434 & ESR1 & $C>T$ & $\begin{array}{l}\text { GRCh37.p13 chr } 6 \\
\text { GRCh38.p12 chr } 6\end{array}$ & $\begin{array}{l}\text { NC_000006.11:g.152358940C>T } \\
\text { NC_000006.12:g.152037805C>T }\end{array}$ \\
\hline rs11742635 & FBN2 & $G>T$ & $\begin{array}{l}\text { GRCh37.p13 chr } 5 \\
\text { GRCh38.p12 chr } 5\end{array}$ & $\begin{array}{l}\text { NC_000005.9:g.127788794G>T } \\
\text { NC_000005.10:g.128453101G>T }\end{array}$ \\
\hline rs12457644 & CELF4 & $G>A$ & $\begin{array}{l}\text { RCh37.p13 chr } 18 \\
\text { GRCh38.p12 chr } 18\end{array}$ & $\begin{array}{l}\text { NC_000018.9:g.34944976 G>A } \\
\text { NC_000018.10:g.37365013G>A }\end{array}$ \\
\hline
\end{tabular}


выше в подгруппе с неотягощенным анамнезом, что делает его фактором риска развития миомы матки, но не связывает с наследственной предрасположенностью. Возможно, данный аллель больше ассоциирован с другими факторами развития данной патологии.

\section{ОБСУЖДЕНИЕ РЕЗУЛЬТАТОВ}

В настоящее время ученые ведут поиск генетических маркеров развития различных социально значимых гинекологических заболеваний для создания генетических панелей ранней диагностики, прогнозирования рецидивирования, оптимизации тактики ведения и создания новых препаратов для лечения данных заболеваний.
Факторы, вызывающие развитие миомы матки, не установлены, хотя научная литература содержит большой объем информации, имеющий отношение к эпидемиологии, генетике, гормональным аспектам и молекулярной биологии этой опухоли. Перечисленные выше фракты определяют актуальность изучения данного заболевания.

Впервые «семейные случаи» миомы матки, а также зависимость частоты и тяжести течения данного заболевания от этнического происхождения женщин были показаны в исследованиях американских генетиков [24-25]. Ученым удалось обнаружить геномные ассоциации с развитием миомы матки при генотипировании 261 женщины европеоидной расы, являющихся членами одной

Таблица 3. Распределение аллелей исследованных полиморфизмов в группе сравнения, в подгруппе Іа (среди женщин с отягощенным анамнезом по данному заболеванию) и в основной группе с миомой матки

\begin{tabular}{|c|c|c|c|c|c|c|c|c|}
\hline \multirow[b]{2}{*}{ ID } & \multirow[b]{2}{*}{$\begin{array}{l}\text { Вариант } \\
\text { аллеля }\end{array}$} & \multicolumn{3}{|c|}{ Частота встречаемости, \% } & \multicolumn{2}{|c|}{ Критерий Фишера } & \multicolumn{2}{|c|}{ OR } \\
\hline & & $\begin{array}{c}\text { Группа } \\
\text { сравнения, } \\
n=30\end{array}$ & $\begin{array}{c}\text { Подгруппа la c } \\
\text { отягощенным } \\
\text { анамнезом, } \\
n=53\end{array}$ & $\begin{array}{c}\text { Основная группа } \\
\text { пациенток с } \\
\text { миомами, } \\
n=100\end{array}$ & $\begin{array}{c}\text { Подруппа la c } \\
\text { отягощенным } \\
\text { анамнезом }\end{array}$ & $\begin{array}{l}\text { Основная } \\
\text { группа с } \\
\text { миомами }\end{array}$ & $\begin{array}{c}\text { Подгруппа la } \\
\text { с отягощенным } \\
\text { анамнезом }\end{array}$ & $\begin{array}{l}\text { Основная } \\
\text { группа } \\
\text { с миомами }\end{array}$ \\
\hline \multirow{3}{*}{$\begin{array}{c}\text { KCNMB2 } \\
\text { rs12637801 }\end{array}$} & AA & 0,00 & 0,02 & 0,02 & \multirow{3}{*}{0,04} & \multirow{3}{*}{0,09} & \multirow{3}{*}{2,63} & \multirow{3}{*}{2,01} \\
\hline & $A C$ & 0,47 & 0,23 & 0,28 & & & & \\
\hline & $\mathrm{CC}$ & 0,53 & 0,75 & 0,70 & & & & \\
\hline \multirow{3}{*}{$\begin{array}{c}\text { CELF4 } \\
\text { rs2861221 }\end{array}$} & $\mathrm{CC}$ & 0,60 & 0,71 & 0,66 & \multirow{3}{*}{0,30} & \multirow{3}{*}{0,32} & \multirow{3}{*}{1,64} & \multirow{3}{*}{0,48} \\
\hline & CG & 0,30 & 0,23 & 0,29 & & & & \\
\hline & GG & 0,10 & 0,06 & 0,05 & & & & \\
\hline \multirow{3}{*}{$\begin{array}{c}\text { ESR1 } \\
\text { rs3020434 }\end{array}$} & $\mathrm{CC}$ & 0,43 & 0,61 & 0,58 & \multirow{3}{*}{0,11} & \multirow{3}{*}{0,17} & \multirow{3}{*}{2,09} & \multirow{3}{*}{1,77} \\
\hline & CT & 0,50 & 0,33 & 0,35 & & & & \\
\hline & $\pi$ & 0,07 & 0,06 & 0,07 & & & & \\
\hline \multirow{3}{*}{$\begin{array}{c}F B N 2 \\
\text { rs11742635 }\end{array}$} & $\mathrm{GG}$ & 0,57 & 0,67 & 0,73 & \multirow{3}{*}{0,27} & \multirow{3}{*}{0,09} & \multirow{3}{*}{0,27} & \multirow{3}{*}{2,04} \\
\hline & GT & 0,37 & 0,31 & 0,25 & & & & \\
\hline & $\pi$ & 0,06 & 0,02 & 0,02 & & & & \\
\hline \multirow{3}{*}{$\begin{array}{c}\text { CELF4 } \\
\text { rs12457644 }\end{array}$} & AA & 0,13 & 0,06 & 0,05 & \multirow{3}{*}{0,04} & \multirow{3}{*}{0,09} & \multirow{3}{*}{2,63} & \multirow{3}{*}{2,01} \\
\hline & AG & 0,33 & 0,19 & 0,25 & & & & \\
\hline & $\mathrm{GG}$ & 0,53 & 0,75 & 0,70 & & & & \\
\hline
\end{tabular}

Таблица 4. Распределение аллелей исследованных полиморфизмов в группе сравнения и подгруппе Іб (среди женщин с неотягощенным анамнезом по данному заболеванию) и в основной группе с миомой матки

\begin{tabular}{|c|c|c|c|c|c|c|c|c|}
\hline \multirow[b]{2}{*}{ ID } & \multirow[b]{2}{*}{$\begin{array}{l}\text { Вариант } \\
\text { аллеля }\end{array}$} & \multicolumn{3}{|c|}{ Частота встречаемости, \% } & \multicolumn{2}{|c|}{ Критерий Фишера } & \multicolumn{2}{|c|}{ OR } \\
\hline & & $\begin{array}{c}\text { Группа } \\
\text { сравнения, } \\
n=30\end{array}$ & $\begin{array}{c}\text { Подруппа Іб с } \\
\text { неотягощенным } \\
\text { анамнезом } \\
n=47\end{array}$ & $\begin{array}{c}\text { Основная группа } \\
\text { пациенток с } \\
\text { миомами, } \\
n=100\end{array}$ & $\begin{array}{c}\text { Подгруппа Іб с } \\
\text { неотягощенным } \\
\text { анамнезом }\end{array}$ & $\begin{array}{l}\text { Основная } \\
\text { группа с } \\
\text { миомами }\end{array}$ & $\begin{array}{c}\text { Подгруппа Іб с } \\
\text { неотягощенным } \\
\text { анамнезом }\end{array}$ & $\begin{array}{l}\text { Основная } \\
\text { группа } \\
\text { с миомами }\end{array}$ \\
\hline \multirow{3}{*}{$\begin{array}{c}\text { KCNMB2 } \\
\text { rs12637801 }\end{array}$} & AA & 0,00 & 0,02 & 0,02 & \multirow{3}{*}{0,35} & \multirow{3}{*}{0,09} & \multirow{3}{*}{1,54} & \multirow{3}{*}{2,01} \\
\hline & $\mathrm{AC}$ & 0,47 & 0,34 & 0,28 & & & & \\
\hline & $\mathrm{CC}$ & 0,53 & 0,64 & 0,70 & & & & \\
\hline \multirow{3}{*}{$\begin{array}{c}\text { CELF4 } \\
\text { rs2861221 }\end{array}$} & $\mathrm{CC}$ & 0,60 & 0,60 & 0,66 & \multirow{3}{*}{0,30} & \multirow{3}{*}{0,32} & \multirow{3}{*}{1,64} & \multirow{3}{*}{0,48} \\
\hline & CG & 0,30 & 0,36 & 0,29 & & & & \\
\hline & GG & 0,10 & 0,04 & 0,05 & & & & \\
\hline \multirow{3}{*}{$\begin{array}{c}\text { ESR1 } \\
\text { rs3020434 }\end{array}$} & $\mathrm{CC}$ & 0,43 & 0,53 & 0,58 & \multirow{3}{*}{0,40} & \multirow{3}{*}{0,17} & \multirow{3}{*}{2,83} & \multirow{3}{*}{1,77} \\
\hline & CT & 0,50 & 0,38 & 0,35 & & & & \\
\hline & $\mathrm{TT}$ & 0,07 & 0,09 & 0,07 & & & & \\
\hline \multirow{3}{*}{$\begin{array}{c}\text { FBN2 } \\
\text { rs11742635 }\end{array}$} & GG & 0,57 & 0,79 & 0,73 & \multirow{3}{*}{0,04} & \multirow{3}{*}{0,09} & \multirow{3}{*}{2,83} & \multirow{3}{*}{2,04} \\
\hline & GT & 0,37 & 0,19 & 0,25 & & & & \\
\hline & $\mathrm{TT}$ & 0,06 & 0,02 & 0,02 & & & & \\
\hline \multirow{3}{*}{$\begin{array}{c}\text { CELF4 } \\
\text { rs12457644 }\end{array}$} & AA & 0,13 & 0,79 & 0,05 & \multirow{3}{*}{0,15} & \multirow{3}{*}{0,09} & \multirow{3}{*}{0,29} & \multirow{3}{*}{2,01} \\
\hline & $A G$ & 0,33 & 0,19 & 0,25 & & & & \\
\hline & GG & 0,53 & 0,02 & 0,70 & & & & \\
\hline
\end{tabular}


семьи (первая линия родства). Иммуногистохимическое и генетическое исследования данной группы пациенток выявили аллель риска развития миомы матки - ген FASN, кодирующий синтазу жирных кислот, локализованный в участке 17q25.3 [24-25].

В полногеномном исследовании было проанализировано 457044 SNP у 1607 женщин с клинически диагностированной миомой матки и 1428 женщин группы контроля [26]. SNP с высокой ассоциацией $\left(p<5 \cdot 10^{-5}\right)$ были дополнительно генотипированы у 3466 пациенток с миомой матки и 3245 из группы контроля без миомы матки в истории болезни. Значимые ассоциации с миомой матки для всего генома были выявлены на трех локусах хромосом 10q24.33, 22q13.1 и 11p15.5. Наиболее значимыми при комбинированном анализе в каждом из этих локусов оказались rs7913069 ( $p=8,65 \cdot 10^{-14}$, отношение шансов $(\mathrm{OR})=1,47), \mathrm{rs} 12484776\left(p=2,79 \cdot 10^{-12}\right.$, $\mathrm{OR}=1,23)$ и rs2280543 $\left(p=3,82 \cdot 10^{-12}, \mathrm{OR}=1,39\right)$ соответственно, дальнейшее изучение которых, по мнению авторов, может способствовать выявлению причин развития миомы матки.

В 2017-2018 гг. в пилотном исследовании с целью поиска генетических маркеров развития миом было проведено генотипирование образцов по 906600 SNP пациенток с миомой матки, с выявленной в анамнезе отягощенностью по данному заболеванию (миома матки у родственниц первой линии родства по материнской линии) и контрольной группы, в которую вошли женщины без миомы матки в анамнезе [27]. В результате генотипирования удалось выявить шесть полиморфизмов (rs3020434, rs11742635, rs124577644, rs12637801, rs2861221, rs17677069 генов ESR1, FBN2, CELF4, KCWMB2), частоты которых статистически различались в обеих исследуемых группах по сравнению с остальными SNP, при этом были больше в группе женщин с отягощенным анамнезом по сравнению с группой контроля. Дальнейшее исследование рассматривало только указанные выше шесть полиморфизмов, которые, вероятно, связаны с развитием миомы матки. В этой работе не было обнаружено редких аллелей полиморфизмов генов rs3020434, rs11742635, rs2861221, и rs17677069 в группах с отягощенным анамнезом [27].

В нашем исследовании проведено генотипирование образцов по 5 SNP (rs12637801, rs2861221, rs3020434, rs11742635, rs12457644) пациенток с отягощенным анамнезом, у которых миома была диагностирована у ближайших родственниц по материнской линии (мама, бабушка, сестра, тетя) - подгруппа Іа, пациенток без отягощенного анамнеза - подгруппа Іб и пациенток группы сравнения без миомы матки в анамнезе. Согласно полученным данным, генотипическая частота встречаемости аллеля С однонуклеотидного полиморфизма в гене KCWMB2 (rs12637801) статистически значимо выше в подгруппе пациенток с отягощенным анамнезом по сравнению с группой сравнения (87\% против 77\%; $p=0,04 ; \mathrm{OR}=2,63)$ и генотипическая частота встречаемости аллеля $\mathrm{G}$ однонуклеотидного полиморфизма в гене CELF4 (rs124577644) статистически значимо выше в подгруппе пациенток с отягощенным анамнезом по сравнению с группой сравнения (85\% против 0,70\%; $p=0,04 ; \mathrm{OR}=2,63)$ соответственно. Наличие данных аллелей может быть связано с повышенным риском развития миомы матки и являться наследственной предрасположенностью к развитию данного заболевания.

\section{ВЫВОДЬ}

В данной работе была выявлена ассоциация полиморфизмов генов rs12637801, rs2861221, rs3020434, rs11742635, rs12457644 с отягощенностью анамнеза пациенток по миоме матки и оценена зависимость риска развития данного заболевания от генотипа по исследованным генетическим локусам. Возможно, наша тактика исследования полиморфизмов генов сможет объяснить механизмы формирования «семейных случаев» миомы матки и поможет создать генетическую диагностическую панель для оценки степени риска развития лейомиомы. Направлением дальнейших исследований является генотипирование родственниц пациенток, имеющих семейный анамнез для подтверждения связи заболевания с выявленными генетическими маркерами.

\section{Литература}

1. Адамян Л. В., Андреева Е. Н., Артымук Н. В., Белоцерковцева Л. Д., Беженарь В. Ф., Геворкян М. А. и др. Миома матки: диагностика, лечение и реабилитация. 2015; 101.

2. Адамян Л. В. Спицын В. А., Андреева Е. Н. Генетические акпекты гинекологических заболеваний. М.: ГЭОТАР-Медиа, 2008; 215.

3. Согоян Н. С., Кузнецова М. В., Асатурова А. В., Адамян Л. В., Трофимов Д. Ю. Соматические мутации в экзоне 2 гена MED12 у женщин с одиночной и множественной миомой матки. Акушерство и гинекология. 2018; (12): 63-70.

4. Согоян Н. С., Адамян Л. В. Генетические механизмы развития миомы матки. Проблемы репродукции. 2016; 22 (1): 28-34.

5. Кудрина Е. А., Бабурин Д. В. Миома матки: современные аспекты патогенеза и лечения (клиническая лекция). Архив акушерства и гинекологии им. В. Ф. Снегирева. 2016; 3 (1): 4-10.

6. Кузнецова М. В., Трофимов Д. Ю., Тихончук Е. Ю., Согоян Н. С., Адамян Л. В., Сухих Г. Т. Молекулярные механизмы патогенеза миомы матки: анализ мутаций гена MED12 в Российской популяции. Акушерство и гинекология. 2016; (10): 85-90.

7. Сидорова И. С. Миома матки: возможности лечения и просилактики. Русский медицинский журнал. (Спец. номер:

Мать и дитя.) 2002; 10 (7): 336-9.

8. Barjon K, Mikhail LN. Uterine Leiomyomata (Fibroids). StatPearls Publishing, Treasure Island (FL) StatPearls. 2019; 15.

9. Baird DD, Dunson DB, Hill MC, Cousins D, Schectman, JM High cumulative incidence of uterine leiomyoma in black and white women: ultrasound evidence. American journal of obstetrics and gynecology. 2003; 188 (1): 100-107.

10. Gracia M, Carmona F. Uterine myomas: clinical impact and pathophysiological bases. European Journal of Obstetrics and Gynecology and Reproductive Biology. 2020; (1): 1-5.

11. Тихомиров А. Л. Патогенетическое обоснование ранней диагностики, лечения и профилактики миомы матки [диссертация]. М., 1998.

12. Тихомиров А. Л. Миома, патогенетическое обоснование органосохраняющего лечения. М.: Медицина, 2013; 319.

13. Brouwer MW, Tebbe-Gholami M, Starink MV. Hereditary leiomyomatosis: a woman with red-brown nodules. Nederlands tijdschrift voor geneeskunde. 2015; (159): A8867-A8867.

14. Bulun SE. Uterine fibroids. New England Journal of Medicine. 2013; 369 (14): 1344-55.

15. Mäkinen N, Mehine M, Tolvanen J, Kaasinen E, Li Y, Lehtonen HJ, 
et al. MED12, the mediator complex subunit 12 gene, is mutated at high frequency in uterine leiomyomas. Science. 2011; 334 (6053): 252-5.

16. Mittal P, Shin $\mathrm{YH}$, Yatsenko SA, Castro CA, Surti U, Rajkovic A. MED12 gain-of-function mutation causes leiomyomas and genomic instability. The Journal of clinical investigation. 2015; 125 (8): 3280-4.

17. Akbari M, Do AA, Yassaee F, Mirfakhraie R. MED12 Exon 1 Mutational Screening in Iranian Patients with Uterine Leiomyoma. Reports of biochemistry \& molecular biology. 2019; 8 (1): 1-21.

18. Markowski DN, Bartnitzke S, Löning T, Drieschner N, Helmke BM, Bullerdiek J. MED12 mutations in uterine fibroids-their relationship to cytogenetic subgroups.International journal of cancer. 2012; 131 (7): 1528-36.

19. Савицкий Г. А., Савицкий А. Г. Миома матки (проблемь патогенеза и патогенетической терапии). Новое в акушерстве и гинекологии. Санкт-Петербург: ЭЛБИ, 2000; 236.

20. Самойлова Т. Е. Возможности и перспективы медикаментозного лечения миомы матки. Медицинский совет. 2013; (3): 106-9.

21. Пшеничнюк Е. Ю., Кузнецова М. В., Бурменская О. В., Кочеткова Т. О., Непша О. С., Тросимов Д. Ю. и др. Ассоциация между частотами встречаемости однонуклеотидных полиморфизмов в генах ZNF366 и VEZT и риском развития наружного генитального эндометриоза: данные по российской популяции. Акушерство и гинекология. 2017; (6): 64-73.

22. Sakai K, Tanikawa C, Hirasawa A, Chiyoda T, Yamagami W, Kataoka F. Identification of a novel uterine leiomyoma GWAS locus in a Japanese population. Scientific Reports. 2020; 10 (1): 1-8.

23. LaFleur B. Book Review: Statistical methods for rates and proportions. Clinical Trials. 2004; 1 (6): 567-8.

24. Gallagher CS, Makinen N, Harris HR, Uimari O, Cook JP, Shigesi N, et al. Genome-wide association analysis identifies 27 novel loci associated with uterine leiomyomata revealing common genetic origins with endometriosis. Biorxiv. 2018; 1-26.

25. Gallagher CS, Mäkinen N, Harris HR, RahmiogluN, UimariO, Cook JP, et al. Genome-wide association and epidemiological analyses reveal common genetic origins between uterine leiomyomata and endometriosis. Nature communications. 2019; 10 (1): 1-11.

26. Cha PC, Takahashi A, Hosono N, Low SK, Kamatani N, Kubo M, et al. A genome-wide association study identifies three loci associated with susceptibility to uterine fibroids. Nature genetics. 2011; 43 (5): 447

27. Согоян Н. С., Кузнецова М. В., Лоломадзе Е. А., Михайловская Г. В., Мишина Н. Д., Трофимов Д.Ю., и др. Исследование полиморсиизмов rs3020434, rs11742635, rs124577644, rs12637801, rs2861221, rs17677069 у женщин с миомой матки и отягощенным анамнезом. Акушерство и гинекология. 2019; (10): 115-28.

\section{References}

1. Adamyan LV, Andreeva EN, Artymuk NV, Belocerkovceva LD, Bezhenar VF, Gevorkyan MA, i dr. Mioma matki: diagnostika, lechenie i reabilitacija. 2015; 101. Russian.

2. Adamyan LV, Spicyn VA, Andreeva EN. Geneticheskie akpekty ginekologicheskih zabolevanij. M., GJeOTAR-Media, 2008; 215. Russian.

3. Sogoyan NS, Kuznetsova MV, Asaturova AV, Adamyan LV, Trofimov DYu. Somaticheskie mutacii v jekzone 2 gena MED12 u zhenshhin s odinochnoj i mnozhestvennoj miomoj matki. Akusherstvo i ginekologija. 2018; (12): 63-70. Russian.

4. Sogoyan NS, Adamyan LV. Geneticheskie mehanizmy razvitija miomy matki. Problemy reprodukcii. 2016; 22 (1): 28-34. Russian.

5. Kudrina EA, Baburin DV. Mioma matki: sovremennye aspekty patogeneza i lechenija (klinicheskaja lekcija). Arhiv akusherstva ginekologii im. VF Snegireva. 2016; 3 (1): 4-10. Russian.

6. Kuznetsova MV, Trofimov DYu, Tihonchuk EYu, Sogoyan NS, Adamyan LV, Suhih GT. Molekuljarnye mehanizmy patogeneza miomy matki: analiz mutacij gena MED12 v Rossijskoj populjacii. Akusherstvo i ginekologija. 2016; (10): 85-90. Russian.

7. Sidorova IS. Mioma matki: vozmozhnosti lechenija i profilaktiki. Russkij medicinskij zhurnal. (Spec. nomer: Mat' i ditja.) 2002; 10 (7): 336-9. Russian.

8. Barjon K, Mikhail LN. Uterine Leiomyomata (Fibroids). StatPearls Publishing, Treasure Island (FL) StatPearls. 2019; 15.

9. Baird DD, Dunson DB, Hill MC, Cousins D, Schectman, JM High cumulative incidence of uterine leiomyoma in black and white women: ultrasound evidence. American journal of obstetrics and gynecology. 2003; 188 (1): 100-107.

10. Gracia M, Carmona F. Uterine myomas: clinical impact and pathophysiological bases. European Journal of Obstetrics and Gynecology and Reproductive Biology. 2020; (1): 1-5.

11. Tihomirov AL. Patogeneticheskoe obosnovanie rannej diagnostiki, lechenija i profilaktiki miomy matki [dissertacija]. M., 1998. Russian.

12. Tihomirov AL. Mioma, patogeneticheskoe obosnovanie organosohranjajushhego lechenija. M.: Medicina, 2013; 319. Russian.

13. Brouwer MW, Tebbe-Gholami M, Starink MV. Hereditary leiomyomatosis: a woman with red-brown nodules. Nederlands tijdschrift voor geneeskunde. 2015; (159): A8867-A8867.

14. Bulun SE. Uterine fibroids. New England Journal of Medicine. 2013: 369 (14): 1344-55.

15. Mäkinen N, Mehine M, Tolvanen J, Kaasinen E, Li Y, Lehtonen HJ, et al. MED12, the mediator complex subunit 12 gene, is mutated at high frequency in uterine leiomyomas. Science. 2011; 334
(6053): 252-5.

16. Mittal P, Shin YH, Yatsenko SA, Castro CA, Surti U, Rajkovic A. MED12 gain-of-function mutation causes leiomyomas and genomic instability.The Journal of clinical investigation. 2015; 125 (8): 3280-4.

17. Akbari M, Do AA, Yassaee F, Mirfakhraie R. MED12 Exon 1 Mutational Screening in Iranian Patients with Uterine Leiomyoma. Reports of biochemistry \& molecular biology. 2019; 8 (1): 1-21.

18. Markowski DN, Bartnitzke S, Löning T, Drieschner N, Helmke BM, Bullerdiek J. MED12 mutations in uterine fibroids - their relationship to cytogenetic subgroups. International journal of cancer. 2012; 131 (7): 1528-36.

19. Savickij GA, Savickij AG. Mioma matki (problemy patogeneza patogeneticheskoj terapii). Novoe $v$ akusherstve i ginekologii. Sankt-Peterburg: JeLBI, 2000; 236. Russian.

20. Samojlova TE. Vozmozhnosti i perspektivy medikamentoznogo lechenija miomy matki. Medicinskij sovet. 2013; (3): 106-9. Russian.

21. Pshenichnjuk EYu, Kuznecova MV, Burmenskaja OV, Kochetkova TO, Nepsha OS, Trofimov DYu, i dr. Associacija mezhdu chastotami vstrechaemosti odnonukleotidnyh polimorfizmov v genah ZNF366 i VEZT i riskom razvitija naruzhnogo genital'nogo jendometrioza: dannye po rossijskoj populjacii. Akusherstvo i ginekologija. 2017; (6): 64-73. Russian.

22. Sakai K, Tanikawa C, Hirasawa A, Chiyoda T, Yamagami W, Kataoka F. Identification of a novel uterine leiomyoma GWAS locus in a Japanese population. Scientific Reports. 2020; 10 (1): 1-8.

23. LaFleur B. Book Review: Statistical methods for rates and proportions. Clinical Trials. 2004; 1 ( 6): 567-8.

24. Gallagher CS, Makinen N, Harris HR, Uimari O, Cook JP, Shigesi N, et al. Genome-wide association analysis identifies 27 novel loci associated with uterine leiomyomata revealing common genetic origins with endometriosis. Biorxiv. 2018; 1-26.

25. Gallagher CS, Mäkinen N, Harris HR, RahmiogluN, UimariO, Cook JP, et al. Genome-wide association and epidemiological analyses reveal common genetic origins between uterine leiomyomata and endometriosis. Nature communications. 2019; 10 (1): 1-11.

26. Cha PC, Takahashi A, Hosono N, Low SK, Kamatani N, Kubo M, et al. A genome-wide association study identifies three loci associated with susceptibility to uterine fibroids. Nature genetics. 2011; 43 (5): 447

27. Sogoyan NS, Kuznetsova MV, Lolomadze EA, Mikhailovskaya GV, Mishina ND, Trofimov DYu, et al. A study of polymorphisms rs3020434, rs 1742635, rsl24577644, rsl2637801, rs2861221, and rs 17677069 in women with uterine leiomyomas and a family history of the disease. Obstetrics and Gynegology. 2019; (10): 115-28. 